\title{
Tumor cell invasion from the marginal sinus into extranodal veins during early-stage lymph node metastasis can be a starting point for hematogenous metastasis
}

\author{
Tetsuya Kodama ${ }^{1,2}$, Shiro Mori ${ }^{1,2,3}$, Masato Nose ${ }^{4}$ \\ ${ }^{1}$ Graduate School of Biomedical Engineering, Tohoku University, Sendai 980-8575, Japan. \\ 2Biomedical Engineering Cancer Research Center, Graduate School of Biomedical Engineering, Tohoku University, Sendai 980 - \\ 8575, Japan. \\ ${ }^{3}$ Department of Oral and Maxillofacial Surgery, Tohoku University Hospital, Sendai 980-8575, Japan. \\ ${ }^{4}$ Institute for Promotion of Advanced Science and Technology, Ehime University, Matsuyama 790-8577, Japan.
}

Correspondence to: Prof. Tetsuya Kodama, Graduate School of Biomedical Engineering, Tohoku University, 4-1 Seiryo, Aoba, Sendai 980-8575, Japan.E-mail: kodama@tohoku.ac.jp

How to cite this article: Kodama T, Mori S, Nose M. Tumor cell invasion from the marginal sinus into extranodal veins during early-stage lymph node metastasis can be a starting point for hematogenous metastasis. J Cancer Metastasis Treat 2018;4:56. http://dx.doi.org/10.20517/2394-4722.2018.61

Received: 10 Sep 2018 First Decision: 7 Oct 2018 Revised: 20 Oct 2018 Accepted: 29 Oct 2018 Published: 9 Nov 2018

Science Editor: William Schiemann Copy Editor: Cai-Hong Wang Production Editor: Zhong-Yu Guo

\begin{abstract}
Aim: To investigate whether tumor cells in a lymph node (LN) can invade from the marginal sinus into extranodal veins via vessel branches that communicate with intranodal veins and whether this can be a starting point for hematogenous metastasis at the early stage of LN metastasis.
\end{abstract}

Methods: Vascular and lymphatic networks of LNs in MXH10/Mo-/pr//pr mice were investigated using threedimensional micro-computed tomography and histological methods. Flow in the blood vessel networks of LNs was investigated by fluorescence microscopy. Tumor cells were injected into the subiliac LNs of MXH1O/MoIpr/lpr mice to induce metastasis to the proper axillary LNs. Tumor development in the proper axillary LN was detected using an in vivo bioluminescence imaging system. A two-dimensional image of the proper axillary LN microvasculature was reconstructed using a contrast-enhanced high-frequency ultrasound system.

Results: Extranodal veins communicated with intranodal veins via branches that penetrated the capsule, and blood flowed from intranodal veins to extranodal veins. Tumor cells that had metastasized to the marginal sinus invaded these communicating veins to develop hematogenous metastases.

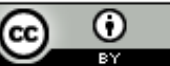

(C) The Author(s) 2018. Open Access This article is licensed under a Creative Commons Attribution 4.0 International License (https://creativecommons.org/licenses/by/4.0/), which permits unrestricted use sharing, adaptation, distribution and reproduction in any medium or format, for any purpose, even commercially, as long as you give appropriate credit to the original author(s) and the source, provide a link to the Creative Commons license, and indicate if changes were made.

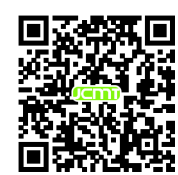


Conclusion: Metastatic LNs that would be considered by clinical imaging to be stage NO can be a starting point for hematogenous metastasis. The study findings highlight the need for the development of novel techniques for the diagnosis and treatment of early-stage LN metastasis, i.e., when standard diagnostic imaging might incorrectly classify the LN as stage NO.

Keywords: Lymph node, metastasis, NO, lymph node-mediated hematogenous metastasis

\section{INTRODUCTION}

Tumor cells reach the marginal sinus of a sentinel lymph node (LN) via afferent lymphatic vessels, after which they proliferate along the lymphoid sinus, invade the cortex and reach the medulla ${ }^{[1]}$. The abundant vascular network in a $\mathrm{LN}^{[2]}$ allows tumor cells to grow without the induction of tumor neovasculature ${ }^{[3,4]}$. Since tumor cells growing in a LN can infiltrate both the lymphatic channel and the vascular network, a sentinel LN can be the origin of lymphatic metastasis to downstream LNs as well as hematogenous metastasis $^{[5]}$. It has been suggested that high endothelial venules (HEVs) may be involved in the mechanisms underlying systemic metastasis ${ }^{[5,6]}$, but the details remain unknown. Clinically, a LN is judged as positive for metastasis ( $>\mathrm{N} 1$ ) if tumor invasion is detected by diagnostic imaging or aspiration cytology. However, since a LN can be erroneously classified as stage No during the early stages of tumor invasion, a false-No LN can potentially be a source of systemic metastasis. For example, in the NSABP-32 trial ${ }^{[7]}$, patients with breast cancer judged incorrectly to be stage No had no difference in overall survival, diseasefree survival and distant disease-free interval to patients judged to be stage No. In other words, tumor cells may have undergone systemic metastasis at a stage when LNs were incorrectly classified as No.

Recently, we demonstrated that a fluorescent dye injected locally into a LN flowed into both the efferent lymphatic vessel and extranodal veins and that intranodal and extranodal veins communicated via branches that passed through the capsule ${ }^{[8,9]}$, a feature not described in conventional textbooks of anatomy. Thus, tumor cells can undergo both lymphatic and hematogenous metastasis. Based on these results, we proposed a theory of LN-mediated hematogenous metastasis, whereby LNs can be the origin of systemic metastasis ${ }^{[8,9]}$.

In this study, we used a mouse model in which metastasis to the proper axillary LN (PALN) was induced by the inoculation of tumor cells into the subiliac LN (SiLN). We found that during early-stage PALN metastasis (confirmed by pathological imaging), the invasion of tumor cells from the marginal sinus into intranodal veins and then extranodal veins may be a first step in the mechanism of hematogenous metastasis from a LN.

\section{METHODS}

Experiments were carried out in accordance with published guidelines and approved by the Institutional Animal Care and Use Committee of Tohoku University.

\section{Mice}

MXH10/Mo-lpr/lpr (MXH10/Mo/lpr) mice (12-17 weeks old), which are a congenic strain of MRL/Mp-lpr/lpr and $\mathrm{C} 3 \mathrm{H} / \mathrm{HeJ}-l p r / l p r$ mice ${ }^{[10]}$, were bred under specific pathogen-free conditions in the Animal Research Institute, Graduate School of Medicine, Tohoku University, Sendai, Japan. The LNs enlarge to about $10 \mathrm{~mm}$ in diameter at 12 weeks of age due to invasion by $l p r-\mathrm{T}\left(\mathrm{CD} 4 \mathrm{CD} 8 \mathrm{~B} 220^{+} \mathrm{Thy}^{+}\right) \mathrm{cells}^{\left[\mathrm{f}^{[1]}\right.}$. The anatomical locations and nomenclatures of murine LNs have often been ignored or assigned incorrectly; in this study, we used the term "subiliac LN" instead of "inguinal LN"[12].

\section{Micro-computed tomography imaging}

Specimens were analyzed using high-resolution micro-computed tomography (micro-CT) scanning (scanXmate/E090, Comscan Tecno). Barium contrast agent (mean size, $935.7 \mathrm{~nm}$ ) was prepared as 
previously described ${ }^{[13]}$. With the mouse under deep general anesthesia, $0.1 \mathrm{~mL}$ heparin (Novo-Heparin, 1000 units $/ \mathrm{mL}$, Mochida Pharmaceutical) was administered intravenously and $0.05 \mathrm{~mL}$ papaverine hydrochloride (40 mg/mL, Nichi-Iko) was given subcutaneously. Ten min later, a syringe pump (Legato100, KD Scientific) was used to infuse $4 \mathrm{~mL}$ of saline $(18 \mathrm{~mL} / \mathrm{h})$ into the left ventricle through a thoracotomy. After cutting of the caudal vena cava and draining of the blood from the body, $4 \mathrm{~mL}$ of contrast medium was injected into the left ventricle $(18 \mathrm{~mL} / \mathrm{h})$ via a T-shaped stopcock. Following perfusion with contrast medium, the LNs were dissected and kept at $4{ }^{\circ} \mathrm{C}$ for $>2 \mathrm{~h}$ to allow fixation to occur. The samples were placed on the stage of a micro-CT scanner (the gutta percha was used as a landmark for positioning) and scanned (angiography) at resolutions of 5-30 $\mu \mathrm{m}$ and a slice thickness of $100 \mu \mathrm{m}$. Acquired slice data were rendered as 3D images using a $3 \mathrm{D}$ analysis suite (Amira, Maxnet).

\section{Cell culture}

KM-Luc/GFP cells (mouse malignant fibrous histiocytoma-like cells derived from an MRL/Mp-gld/gld mouse expressing a fusion of the luciferase and enhanced-green fluorescent protein genes) were cultured as previously described ${ }^{[14]}$. FM3A-Luc cells $(\mathrm{C} 3 \mathrm{H} / \mathrm{He}$ mouse mammary carcinoma cells expressing the luciferase gene $)^{[10]}$ were maintained in RPMI-1640 medium supplemented with $10 \%$ fetal bovine serum, $1 \%$ L-glutamine-penicillin-streptomycin and $1 \mathrm{mg} / \mathrm{mL}$ G418 (Sigma-Aldrich). Both cell types had an $\mathrm{H}-2^{k}$

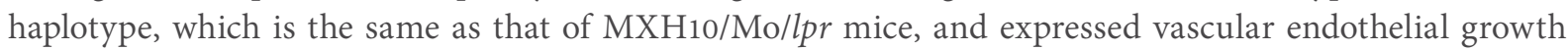
factor (VEGF)-A and VEGF-B but not VEGF-C; KM-Luc/GFP but not FM3A-Luc cells showed slight VEGFD expression ${ }^{[15]}$. The relative growth rates of KM-Luc/GFP and FM3A-Luc cells were 3.8/day and 1.1/day, respectively ${ }^{[8]}$. Cell lines were incubated $\left(37{ }^{\circ} \mathrm{C}, 5 \% \mathrm{CO}_{2} / 95 \% \mathrm{O}_{2}\right)$ until $80 \%$ confluence was achieved. Lack of Mycoplasma contamination was confirmed on the inoculation day (MycoAlert Mycoplasma Detection Kit; Lonza Rockland).

\section{NBD-liposomes}

NBD-liposomes were synthesized from 1,2-distearoyl-sn-glycero-3-phosphatidylcholine (DSPC; MC8080, NOF Co.), 1,2-distearoyl-sn-glycerol-3-phosphatidylethanolamine-methoxy- polyethyleneglycol (DSPEPEG[2000-OMe]; DSPE-020CN, NOF Co.), and 1,2-dipalmitoyl-sn-glycero- 3-phosphoethanolamine-N(7-nitro-2-1,3-benzoxadiazol-4-yl) (NBD-DPPE; FE6060, NOF Co. $)^{[9,16]}$. The size and zeta potential of the NBD-liposomes was $642 \mathrm{~nm}$ and $-1.5 \mathrm{mV}$, respectively, as measured using a particle size and zeta potential analyzer (ELSZ-2; Otsuka Electronics).

\section{Visualization of venous flows internal and external to a $\mathrm{LN}$}

Under deep general anesthesia, an arc-shaped incision was made in the abdominal skin of a mouse $(n=1,12$ weeks old) from the subiliac region to the proper axillary region, and $100 \mu \mathrm{L}$ of $0.01 \mu \mathrm{mol} / \mathrm{L}$ NBD-liposomes was injected into the tail vein $(100 \mu \mathrm{L} / \mathrm{min}, 60 \mathrm{~s})$. Fluid flow in the veins was visualized using a fluorescence stereomicroscope (M165-FC; fluorescence filter: GFP2; excitation: 460-500 nm; emission: > $510 \mathrm{~nm}$; Leica) connected to a high-speed camera (10 frames/s; CoolSNAP HQ2; Photometrics) ${ }^{[8]}$.

\section{Induction of metastasis to the PALN by injection of tumor cells into the SiLN}

KM-Luc/GFP $\left(1.5 \times 10^{6}\right.$ cells $\left./ \mathrm{mL}\right)$ or FM3A-Luc $\left(3.3 \times 10^{5}\right.$ cells $\left./ \mathrm{mL}\right)$ cells (passaged three times) were suspended in a mixture of $20 \mu \mathrm{L}$ phosphate-buffered saline (PBS) and $40 \mu \mathrm{L}$ of $400 \mathrm{mg} / \mathrm{mL}$ Matrigel (Collaborative Biomedical Products). The prepared cells were injected into the center of the SiLN of a mouse (aged 14-16 weeks) using a 27G needle, which was maintained in the same position for 5 min to allow solidification of the Matrigel. Tumor development in the SiLN and metastasis to the PALN (the rates of which depended on the tumor cell type) were detected using an in vivo bioluminescence imaging system (IVIS; Xenogen) at 4 and 7 days post-inoculation of KM-Luc/GFP cells and at 6, 13, 20 and 27 days postinoculation of FM3A-Luc cells. Injection of tumor cells into the SiLN induces metastasis to the PALN via lymphatic vessels as well as hematogenous metastasis via the thoracoepigastric vein $(\mathrm{TEV})^{[9,17]}$; the cell number is highest in the SiLN and second highest in the PALN ${ }^{[14]}$. 


\section{Contrast-enhanced ultrasound imaging and spatiotemporal analysis of pixel intensity variations}

A 2D image of the PALN microvasculature was reconstructed on days 6 and 9 post-inoculation of KMLuc/GFP cells and on days 8, 15, 22 and 29 post-inoculation of FM3A-Luc cells using a contrast-enhanced high-frequency ultrasound (CE-HFUS) system with a 37.5-MHz transducer (RMV-710B, VisualSonics). Each transducer was fixed to a 3D stage control system (Mark-204-MS; Sigma Koki). Contrast images (slice thickness, $100 \mu \mathrm{m}$ ) were captured before and $60 \mathrm{~s}$ after an intravenous bolus injection of $100 \mu \mathrm{L}$ microbubbles (Sonazoid, Daiichi Sankyo) into the tail vein. During imaging, the mouse was positioned on a heated stage and anesthetized with $2 \%$ isoflurane in oxygen ${ }^{[18]}$. Acquired images were analyzed using ultrasound contrast agent-detecting software ${ }^{[19]}$ to measure temporal changes in the PALN microvessel density.

\section{Histological analysis}

PALNs and SiLNs were harvested at the indicated time points, fixed in 10\% formaldehyde in PBS for 3 days, placed on a shaker for 1 day at room temperature and then stored at $4{ }^{\circ} \mathrm{C}$ for 2 days. Next, the samples were dehydrated in $100 \%$ ethanol, placed into a tissue processor, embedded in paraffin and cut into $3-\mu \mathrm{m}$ serial sections using a fully-automated tissue-sectioning device (AS-400, Kurano). Samples were stained with hematoxylin and eosin (HE) or immunostained for detection of CD31-positive vascular endothelial cells.

\section{Statistical analysis}

Data are presented as mean \pm SD or mean \pm SEM. Differences between groups were determined by oneway analysis of variance followed by Tukey's test or an unpaired $t$-test (GraphPad Prism 6J). $P<0.05$ was considered to be statistically significant.

\section{RESULTS}

\section{Connection of the TEV to SiLN blood vessels}

First, we examined the anatomical positions of the PALN, SiLN, accessory axillary LN (AALN) and TEV. An arc-shaped incision was made in the abdominal skin of a mouse from the subiliac region to the proper axillary region [Figure 1AI]. The axillary area contains two LNs, the PALN and AALN ${ }^{[16]}$. The SiLN and AALN lie upstream of the PALN in the lymphatic network. The TEV, which connects the subclavian vein and inferior vena cava, runs adjacent to the PALN [Figure 1AII] and SiLN [Figure 1AIII] and along the lymphatic vessels (not visualized in Figure 1AI) between these LNs. The TEV receives venous blood from the PALN and SiLN via small branches [Figure $1 \mathrm{AII}$ and AIII] ${ }^{[9,16]}$. There were many vessels on the LN that connected to intranodal vessels [Figure 1AII and AIII]. The hilum of the PALN [Figure 1AII] and of the SiLN [Figure 1AIII] was located behind the image.

A series of $3 \mathrm{D}$ micro-CT images, rotated every $60^{\circ}$, revealed a complex vascular network in the PALN [Figure $1 \mathrm{~B}$ ], with many small branches penetrating the LN capsule and connecting the TEV to intranodal blood vessels. There were no similar networks on the reverse side [Figure 1B]. Immunolabeling of CD31 [Figure $1 \mathrm{C}$ ] showed that the TEV ran along and penetrated the capsule of the SiLN [Figure 1CI-IV]. The TEV ran along the capsule (Cap; image I) and connected with intranodal veins penetrating the marginal sinus (Mgs; image II). The branches of TEV ran in the cortex (Cor) under the marginal sinus (image III) and branched into two vessels in the cortex (image IV).

\section{Flow dynamics between the PALN blood vessel network and TEV}

The flow dynamics between the PALN blood vessel network and TEV were visualized under a fluorescence stereomicroscope after intravenous injection of NBD-liposomes [Figure 2]. Consistent with the results shown in Figure 1, the TEV communicated with intranodal blood vessels via many small branches [Figure 2]. In the region excluding the TEV [Figure $2 \mathrm{I}$ and Video 1], a large vein running along the PALN made connections with the intranodal veins, and the combined blood flow drained into the TEV. In the region including the TEV [Figure 2II and Video 2], the venous network inside the LN connected to the TEV. The blood flow 
A

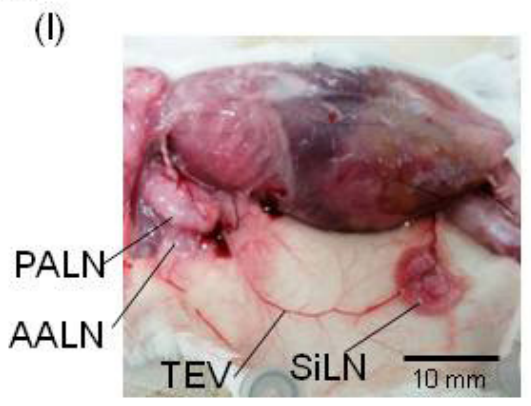

(II) PALN

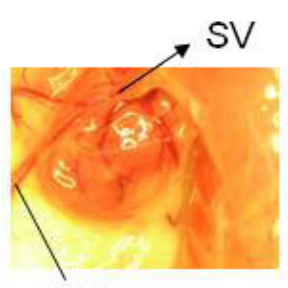

TEV
(III) SiLN

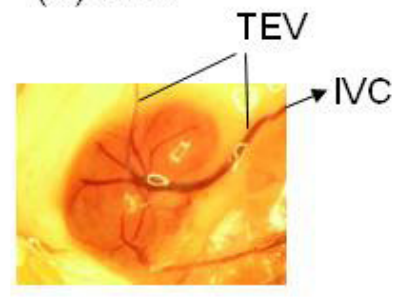

B

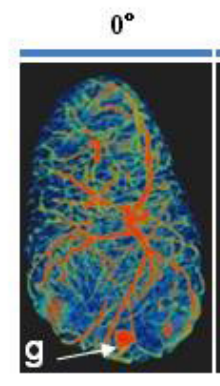

$60^{\circ}$

$120^{\circ}$

$180^{\circ}$

$240^{\circ}$

$300^{\circ}$

C
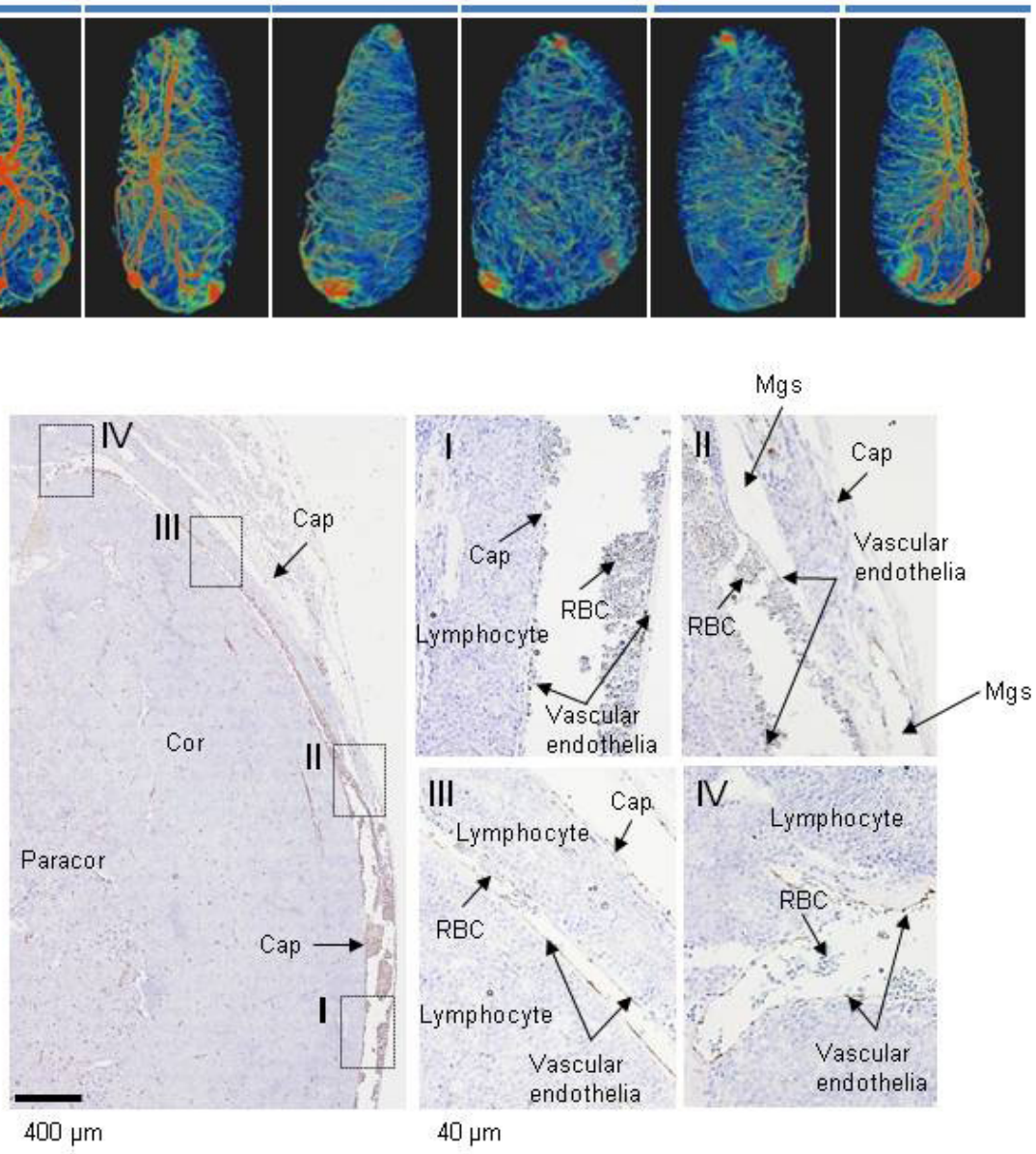

IV

Lymphocyte

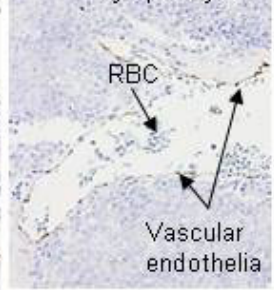

$40 \mu \mathrm{m}$

Figure 1. Vascular and lymphatic networks of lymph nodes (LNs). A: Vascular networks in LNs: (I) a macroscopic view of a 14-weekold mouse (right side) indicating the anatomical positions of the proper axillary LN (PALN), subiliac LN (SiLN), accessory axillary LN (AALN) and thoracoepigastric vein (TEV). The axillary area contains two LNs, the PALN and AALN ${ }^{[16]}$. The SiLN and AALN are upstream of the PALN in the lymphatic network. The TEV, which connects the subclavian vein (SV) and inferior vena cava (IVC), runs adjacent to the SiLN and PALN and along the lymphatic vessels between them (not visualized). The TEV receives venous blood from the SiLN and PALN via small branches; (II) blood vessels running on the PALN in a 17-week-old mouse. There were many vessels on the LN surface. The hilum was behind the image; (III) blood vessels running on the SiLN in a 17-week-old mouse. There were many vessels on the LN surface. The hilum was behind the image: B: three-dimensional micro-computed tomography (micro-CT) images showing the surface and internal vascular structure of the PALN in a 14-week-old mouse. The series of images shows the LN rotated by $60^{\circ}$ each time. The TEV communicated with intranodal blood vessels via many small branches that penetrated the LN capsule. There were no similar networks on the reverse side. Scale: $2 \mathrm{~mm}$. g: gutta-percha; C: images immunostained for CD31 showing the connections between the TEV and SiLN blood vessels in a 16-week-old mouse. Images I-IV (middle and right) are magnified views of the corresponding regions highlighted in the left-most image. The TEV ran along the capsule (image I) and connected with intranodal veins penetrating through the marginal sinus (image II). It is notable that the marginal sinus was extremely close to the intranodal vein. The branches of TEV ran in the cortex under the marginal sinus (image III) and branched into two vessels in the cortex (image IV). RBC: red blood cell; Cap: capsule; Mgs: marginal sinus; Cor: cortex; Paracor: paracortex 


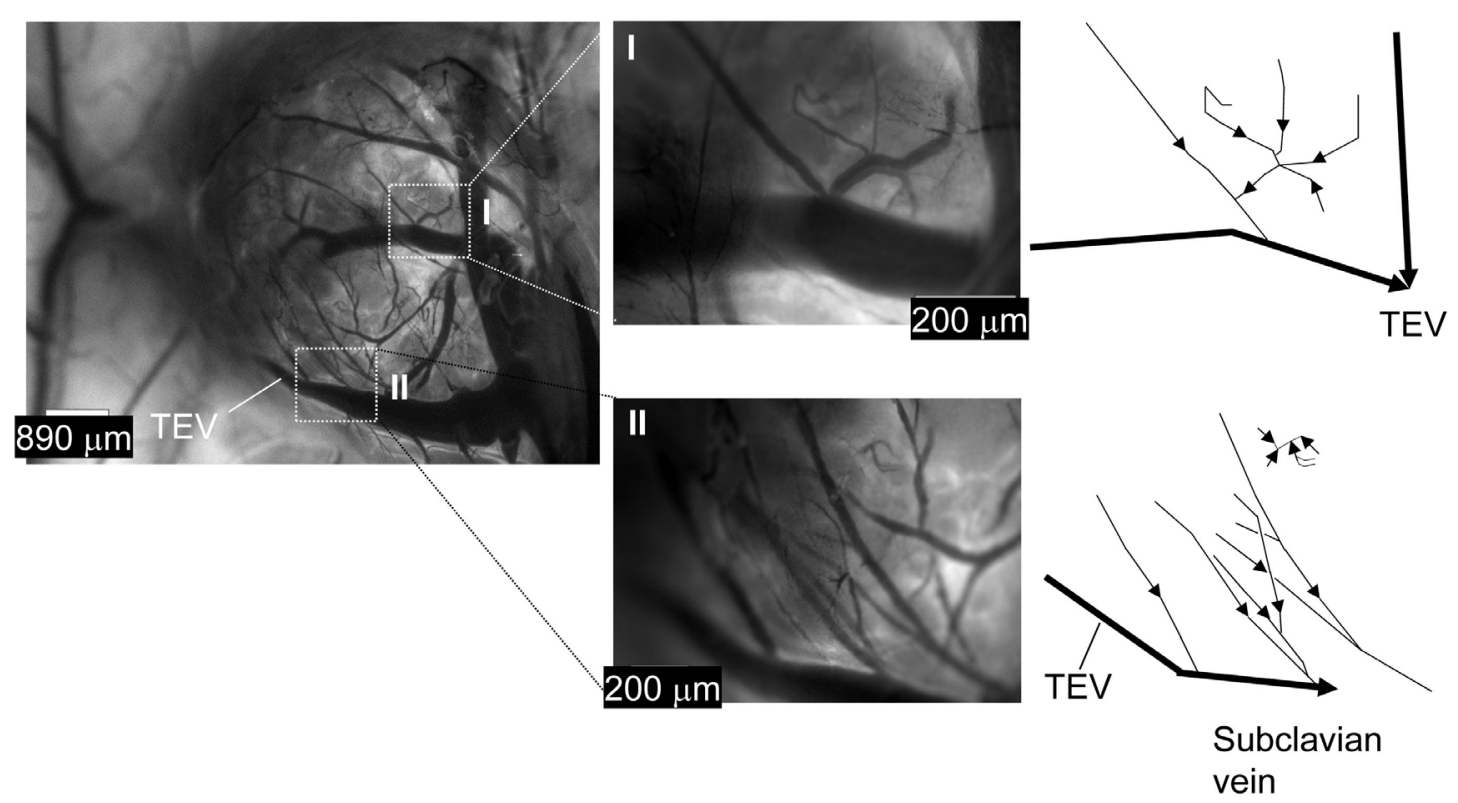

Figure 2. Flow in the blood vessel network of the proper axillary lymph node (PALN; $n=1$ mouse, 12 weeks old). Images I and II are magnified views of the corresponding regions highlighted in the left-most image. Image I shows a region that did not include the thoracoepigastric vein (TEV) and was a frame obtained from Video 1. Image II shows a region that includes the TEV and was a frame obtained from Video 2 . The directions of flow in images I and II are shown schematically to the right of each image

from the TEV enters the subclavian vein ${ }^{[16]}$; since the venous branches from the intranodal veins connect to the TEV, their blood is returned to the venous circulation.

\section{Metastatic SiLN at the false NO stage}

Next, we explored the interaction of tumor cells with intranodal vessels during the early stage of metastasis. Two different tumor cell types were used: KM-Luc/GFP [Figure 3A, C, E] and FM3A-Luc [Figure 3B, D, F]. The injection of tumor cells into the SiLN induced metastasis in the PALN. Luciferase activity in the SiLN and PALN increased with tumor progression for both cell lines (Figure 3A and C for KM-Luc/GFP, Figure 3B and D for FM3A-Luc). Metastasis was detected on day 7 after inoculation of KM-Luc/GFP cells [Figure 3A] and day 27 after inoculation of FM3A-Luc cells [Figure 3B]. Subsequently, we investigated flow dynamics in and around the metastatic PALN using CE-HFUS [Figure $3 \mathrm{E}$ and F]. Microbubbles flowing in the TEV were visualized in experiments using KM-Luc/GFP cells [Figure 3E and Video 3], while confluence of the TEV with other vessels was visualized in experiments using FM3A-Luc cells [Figure 3F and Video 4]. Our previous studies demonstrated no significant change in the volume of the metastatic PALN up to day 7 for KM-Luc cells ${ }^{[20]}$ and day 22 for FM3A-Luc cells ${ }^{[21]}$. Thus, in a clinical setting, the metastatic PALN in these experiments would have been classified as stage No by diagnostic ultrasound.

\section{Interaction of tumor cells with surrounding blood vessels in a LN at the false NO stage}

Histological techniques were used to investigate the interaction of tumor cells in the PALN (at the false No stage) with the surrounding blood vessels at day 6 for KM-Luc/GFP cells [Figure 4A and B] and day 8 for FM3A-Luc cells [Figure 4C-E]. The PALNs were removed after the micro-CT imaging experiments had been completed so that the blood vessels were filled with contrast agent. In experiments using KM-Luc/ GFP cells, serial sections stained with HE [Figure 4A and B, Supplementary Figure 1] or immunostained for CD31 [Supplementary Figure 1] showed an afferent lymphatic vessel entering the LN. Tumor cells from the afferent lymphatic vessel had invaded the marginal sinus as well as vessels that were filled with contrast agent. In experiments using FM3A-Luc cells [Figure 4C and D], metastasized tumor cells were detected in the marginal sinus of the PALN. Importantly, tumor cells that had developed in the marginal sinus had 
A

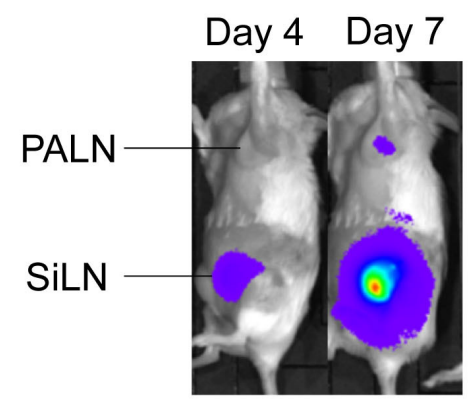

C

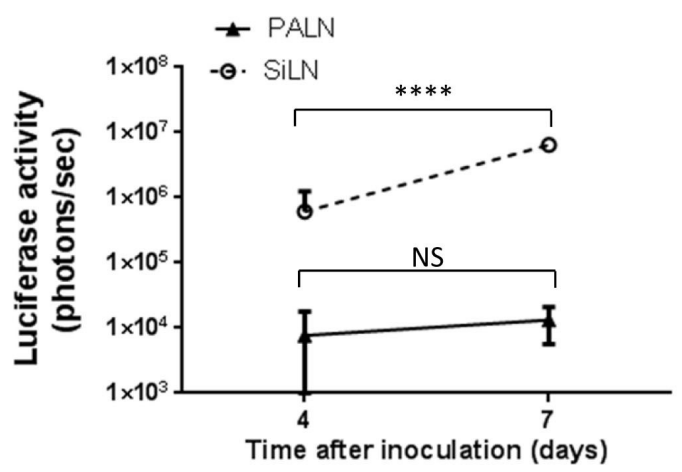

E

Day 6

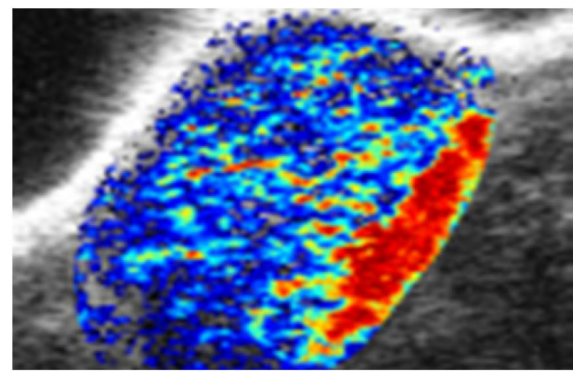

B

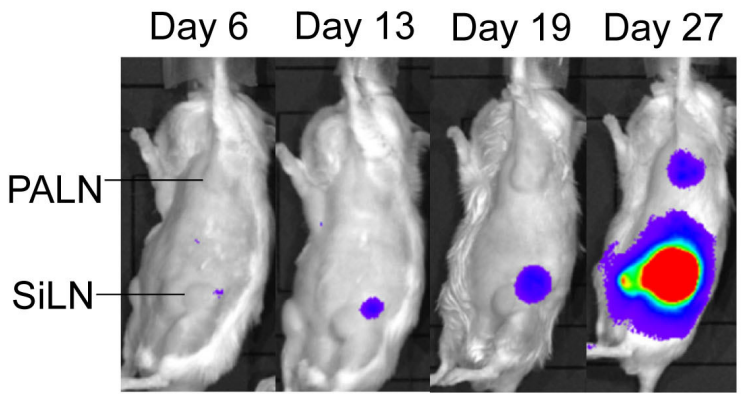

D

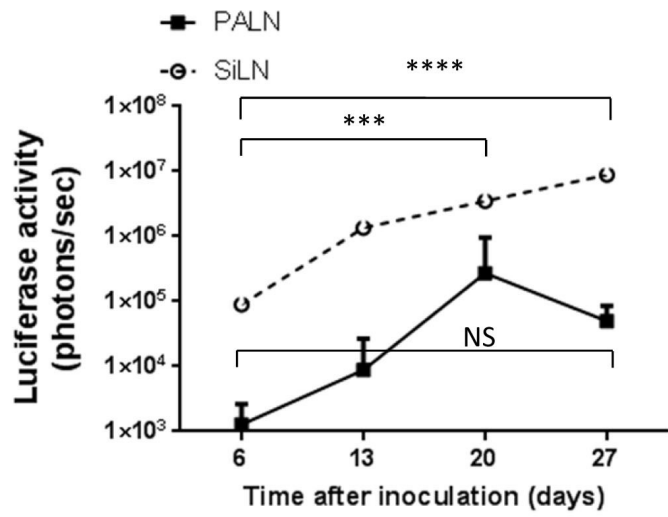

F

Day 8

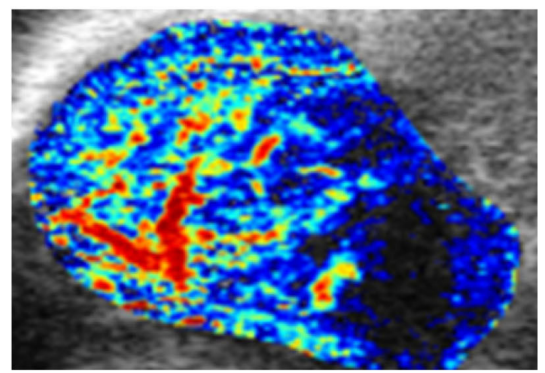

Figure 3. Induction of metastasis of KM-LuC/GFP cells or FM3A-Luc cells to the proper axillary lymph node (PALN) after inoculation of the cells into the subiliac lymph node (SiLN). KM-Luc/GFP cells (A, C, E); FM3A-Luc cells (B, D, F). A: Progression of KM-Luc/GFP cell metastasis from the SiLN to the PALN, as detected from measurements of luciferase activity; B: progression of FM3A-Luc cell metastasis from the SiLN to the PALN, as detected from measurements of luciferase activity; C: quantification of luciferase activity in the SiLN and PALN after inoculation of KM-Luc/GFP cells into the SiLN (SiLN, $n=5$ at day 4 and $n=3$ at day 7; PALN, $n=4$ at day 4 and $n=4$ at day 7 ). ${ }^{* \star \star *} P<0.0001$, unpaired $t$-test. The bars show mean \pm SEM values; D: quantification of luciferase activity in the SiLN and PALN after inoculation of FM3A-Luc cells into the SiLN (SiLN, $n=14$ at day $6, n=10$ at day $13, n=7$ at day 20 and $n=3$ at day 27; PALN, $n=14$ at day $6, n=10$ at day $13, n=7$ at day 20 and $n=3$ at day 27). ${ }^{\star \star \star} P=0.0001,{ }^{\star \star \star \star \star} P<0.0001$, one-way ANOVA and Tukey's test. The bars show mean \pm SEM values; E: representative images of the PALN at day 6 , obtained using contrast-enhanced high-frequency ultrasound (CE-HFUS; $n=5$; Video 3), in a mouse inoculated with KM-Luc/GFP cells. The CE-HFUS image reveals microbubbles flowing in the vessel in the PALN; F: representative images of the PALN at day 8, obtained using CE-HFUS ( $n=5$; Video 4 ), in a mouse inoculated with FM3A-Luc cells. The CE-HFUS image shows microbubbles flowing in the vessel in the PALN. NS: not significant 


\section{KM-Luc/GFP: A, B}

A

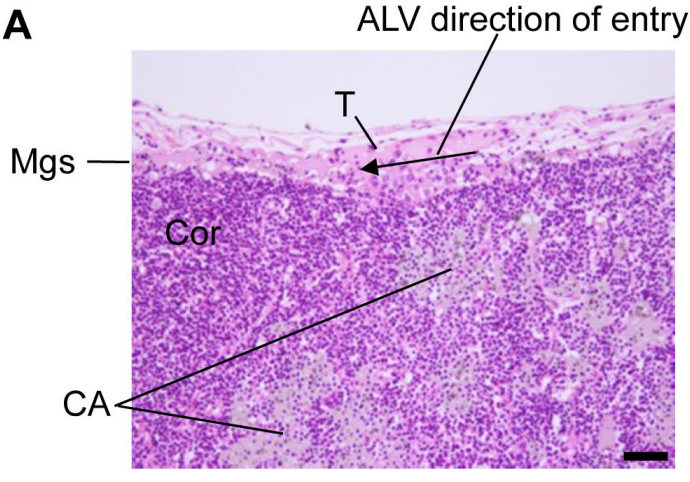

B

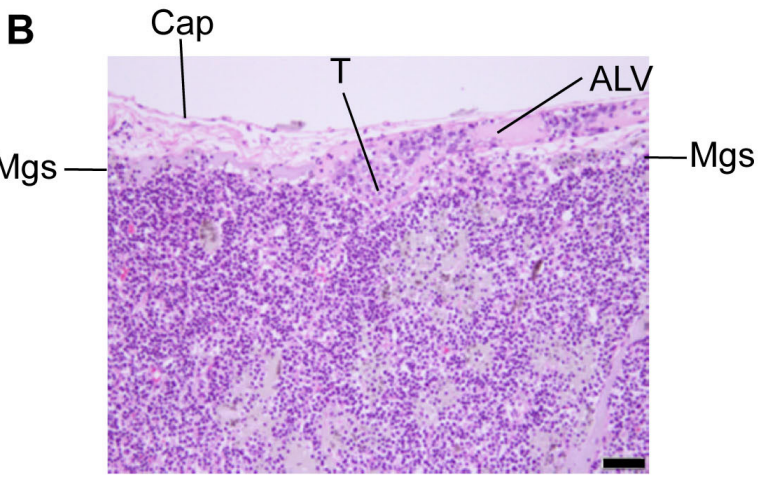

FM3A-Luc: C, D, E
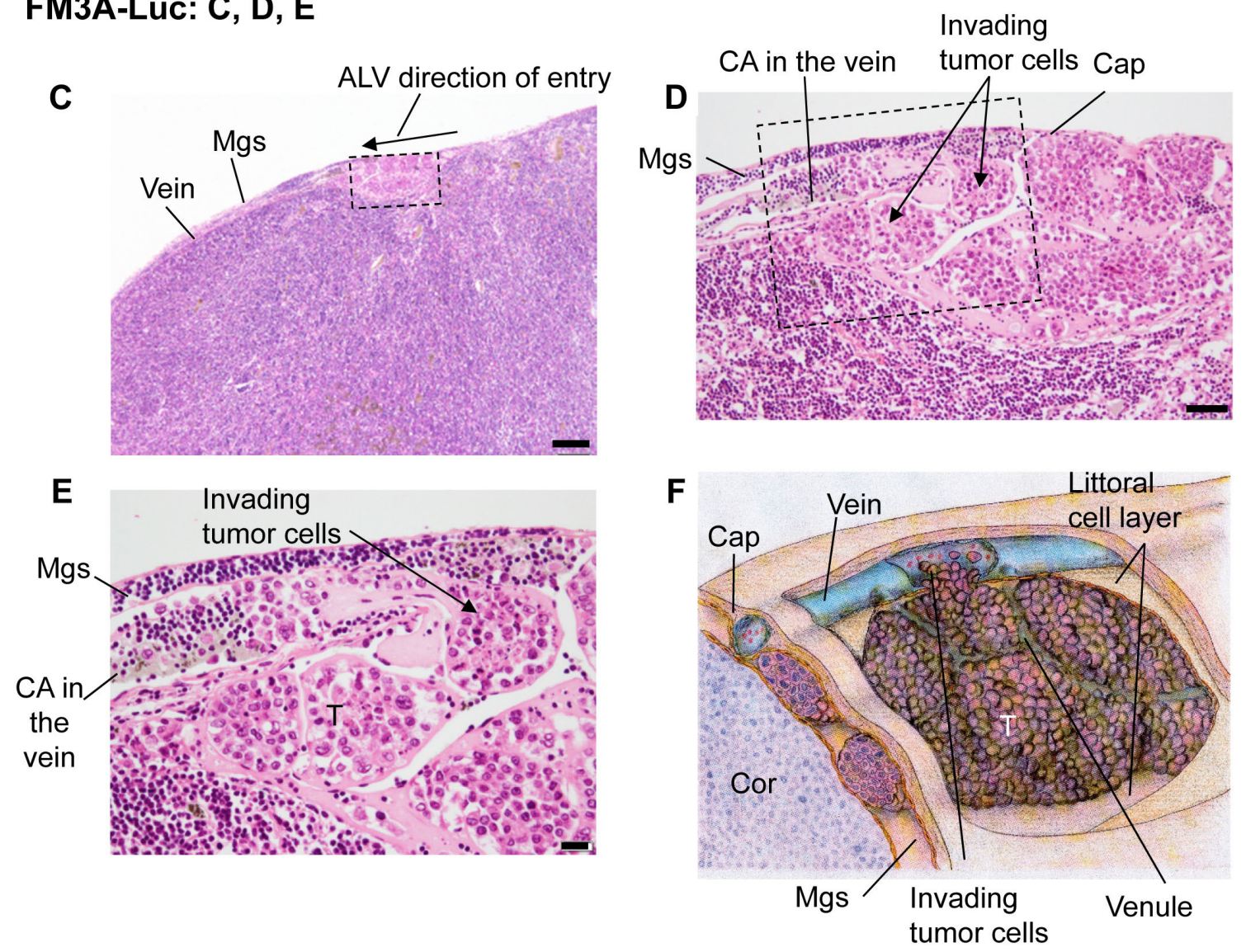

Figure 4. Invasion of tumor cells into a subcapsular vein in the proper axillary lymph node (PALN). A, B: Sections of the PALN at day 6 after inoculation of KM-Luc/GFP cells into the subiliac lymph node (SiLN). The sections were stained with hematoxylin-eosin (HE); the interval between each section was $12 \mu \mathrm{m}$. Tumor cells ( $\mathrm{T}$ ) that had entered from the afferent lymphatic vessel (ALV) had invaded into the vein in the marginal sinus (Mgs) of the PALN. Scale bar: $100 \mu \mathrm{m}$. Cor: cortex; CA: contrast agent; C-F: PALN at day 8 after inoculation of FM3A-Luc cells into the SiLN [C: tumor cells localized in the marginal sinus of the PALN. HE staining. Scale bar: $200 \mu$ m; D: higher magnification of the rectangular region in (C). The marginal sinus was filled with tumor cells in a botryoidal configuration that projected toward the vascular wall. HE staining. Scale bar: $50 \mu \mathrm{m}$. E: higher magnification of the rectangular region in (D). The tumor cells had disrupted the littoral cell layer of the marginal sinus and the vascular wall and had invaded into the vascular lumen (arrow). HE staining. Scale bar: $20 \mu \mathrm{m}$; F: illustration of (D) showing the invasion of metastatic tumor cells from the marginal sinus into the subcapsular vein. Cap: capsule; Cor: cortex; Mgs: marginal sinus (subcapsular lymphatic sinus)] 
entered the region close to the subcapsular vein and invaded the vessel [Figure $4 \mathrm{E}$ ]. The schematic diagram in Figure $4 \mathrm{~F}$ illustrates the internal features of the marginal sinus with metastatic tumor cells invading the subcapsular vein.

\section{DISCUSSION}

The present study demonstrates that tumor cells in the marginal sinus of a LN can invade extranodal veins via branches that communicate with intranodal veins. We suggest that this may be the first step in hematogenous metastasis from LNs. Importantly, this form of metastasis occurs before tumor cells have interfered with well-developed vascular networks and HEVs within the $\mathrm{LN}^{[5,6]}$. Veins are ubiquitously present on the surface layer of the LN $\left[\right.$ Figure 1] ${ }^{[22]}$ and communicate with intranodal veins, allowing blood to flow to the systemic circulation from intranodal vessels. This anatomical arrangement and flow characteristics have not been described previously. Kelch et al. ${ }^{[2]}$ surgically excised individual mesenteric LNs from the mouse and clarified the topology of the entire intranodal vascular network using an automated confocal imaging system and custom-written software. However, unlike the present study, their analysis was limited to the vascular network within the LN. This study utilized malignant fibrous histiocytoma-like KM-Luc/GFP cells and breast cancer FM3A-Luc cells, both of which are syngeneic to the recipient mice. The invasion of tumor cells from the afferent lymphatic vessel into the intranodal veins was confirmed for both cell types [Figure 4, Supplementary Figure 1]. This implies that LN-mediated hematogenous metastasis may be a mechanism relevant to a wide variety of cancer types.

Lymphadenopathy in MXH10/Mo/lpr mice is due to the lpr gene and is characterized by the accumulation of $l p r$-T cells in the paracortical region. The metastatic lesions shown in Figure 4 are located in the marginal sinuses of the LNs. Thus, it is unlikely that there is a direct relationship between abnormal lymphocyte proliferation in the LN parenchyma and tumor cell proliferation and vascular invasion.

MXH10/Mo/lpr mice do not express the fas gene involved in apoptosis, since the lpr gene is a fas-deletion mutant gene. Thus, the immune system in $\mathrm{MXH10/Mo/lpr} \mathrm{mice} \mathrm{is} \mathrm{functional} \mathrm{except} \mathrm{for} \mathrm{the} \mathrm{signaling} \mathrm{path-}$ way related to fas. This precludes us from using human cell lines for our metastasis experiments, which would require immune-deficient mice such as SCID or nude mice. We selected MXH10/Mo/lpr mice for the present experiments because their characteristics make them better suited for use as a model of metastasis than immune-deficient mice implanted with human cell lines.

Fisher et al. ${ }^{[23]}$ reported that LNs were not an effective barrier against tumor cell progression along the lymphatic system based on the observation that tumor cells were confirmed in the efferent lymphatic vessels after their injection into the lymphatic vessels of the rabbit. This means that tumor cells can flow through the lymphatic system via the marginal sinuses of $\mathrm{LNs}^{[17,24]}$, suggesting that lymphatic flow based on the anatomical structure of LNs should be distinguished from the mechanism of tumor metastasis.

Our findings suggest the possibility that tumor cells may undergo hematogenous metastasis at an early stage of LN metastasis, i.e., even before the stage when the infiltration of tumor into the LN is detectable by imaging or aspiration cytology. In other words, false-No metastatic LNs can be a source of hematogenous metastasis. The importance of false-No LNs in distant metastasis is supported by the results of clinical trials suggesting that LN dissection ${ }^{[25,26]}$ and sentinel LN biopsy ${ }^{[27]}$ do not always improve survival in patients with cancer.

The development of new methods to detect and treat metastasis in false-No LNs will be extremely important. In previous studies, we have shown that a lymphatic drug delivery system has great potential in the therapeutic and prophylactic treatment of false-No LNs ${ }^{[25,26]}$, and we expect this technique to be applied in the clinical setting in future. 


\section{DECLARATIONS}

\section{Acknowledgments}

The authors would like to thank Y. Yanagisawa, K. Takeda, W. N. H. W. Hassan and T. Sato for technical assistance and the Biomedical Research Core of Tohoku University Graduate School of Medicine for technical support.

\section{Authors' contributions}

Designed and performed the study, drafted the manuscript and prepared the figures: Kodama $\mathrm{T}$

Interpreted the data, reviewed the manuscript: All authors

\section{Availability of data and materials}

Not applicable.

\section{Financial support and sponsorship}

The study was supported by JSPS KAKENHI [17K20077 (TK) and 17Ho0865 (TK)].

\section{Conflicts of interest}

All authors declared that there are no conflicts of interest.

\section{Ethical approval and consent to participate}

Not applicable.

\section{Consent for publication}

Not applicable.

\section{Copyright}

(C) The Author(s) 2018.

\section{REFERENCES}

1. Starz H, Balda BR, Kramer KU, Buchels H, Wang H. A micromorphometry-based concept for routine classification of sentinel lymph node metastases and its clinical relevance for patients with melanoma. Cancer 2001;91:2110-21.

2. Kelch ID, Bogle G, Sands GB, Phillips AR, LeGrice IJ, et al. Organ-wide 3D-imaging and topological analysis of the continuous microvascular network in a murine lymph node. Sci Rep 2015;5:16534.

3. Jeong HS, Jones D, Liao S, Wattson DA, Cui CH, et al. Investigation of the lack of angiogenesis in the formation of lymph node metastases. J Natl Cancer Inst 2015;107:djv155.

4. Mikada M, Sukhbaatar A, Miura Y, Horie S, Sakamoto M, et al. Evaluation of the enhanced permeability and retention effect in the early stages of lymph node metastasis. Cancer Sci 2017;108:846-52.

5. Qian CN, Berghuis B, Tsarfaty G, Bruch M, Kort EJ, et al. Preparing the "soil": the primary tumor induces vasculature reorganization in the sentinel lymph node before the arrival of metastatic cancer cells. Cancer Res 2006;66:10365-76.

6. Brown M, Assen FP, Leithner A, Abe J, Schachner H, et al. Lymph node blood vessels provide exit routes for metastatic tumor cell dissemination in mice. Science 2018;359:1408-11.

7. Weaver DL, Ashikaga T, Krag DN, Skelly JM, Anderson SJ, et al. Effect of occult metastases on survival in node-negative breast cancer. N Engl J Med 2011;364:412-21.

8. Shao L, Ouchi T, Sakamoto M, Mori S, Kodama T. Activation of latent metastases in the lung after resection of a metastatic lymph node in a lymph node metastasis mouse model. Biochem Biophys Res Commun 2015;460:543-8.

9. Takeda K, Mori S, Kodama T. Study of fluid dynamics reveals direct communications between lymphatic vessels and venous blood vessels at lymph nodes of mice. J Immunol Methods 2017;445:1-9.

10. Shao L, Mori S, Yagishita Y, Okuno T, Hatakeyama Y, et al. Lymphatic mapping of mice with systemic lymphoproliferative disorder: usefulness as an inter-lymph node metastasis model of cancer. J Immunol Methods 2013;389:69-78.

11. Nose M, Komori H, Miyazaki T, Mori S. Genomics of vasculitis: lessons from mouse models. Ann Vasc Dis 2013;6:16-21.

12. Van den Broeck W, Derore A, Simoens P. Anatomy and nomenclature of murine lymph nodes: descriptive study and nomenclatory standardization in BALB/cAnNCrl mice. J Immunol Methods 2006;312:12-9. 
13. Kochi T, Imai Y, Takeda A, Watanabe Y, Mori S, et al. Characterization of the arterial anatomy of the murine hindlimb: functional role in the design and understanding of ischemia models. PLoS One 2013;8:e84047.

14. Li L, Mori S, Sakamoto M, Takahashi S, Kodama T. Mouse model of lymph node metastasis via afferent lymphatic vessels for development of imaging modalities. PLoS One 2013;8:e55797.

15. Miura Y, Mikada M, Ouchi T, Horie S, Takeda K, et al. Early diagnosis of lymph node metastasis: importance of intranodal pressures. Cancer Sci 2016;107:224-32

16. Shao L, Takeda K, Kato S, Mori S, Kodama T. Communication between lymphatic and venous systems in mice. J Immunol Methods 2015;424:100-5.

17. Kodama T, Hatakeyama Y, Kato S, Mori S. Visualization of fluid drainage pathways in lymphatic vessels and lymph nodes using a mouse model to test a lymphatic drug delivery system. Biomed Opt Express 2015;6:124-34.

18. Li XT, Sun YS, Tang L, Cao K, Zhang XY. Evaluating local lymph node metastasis with magnetic resonance imaging, endoluminal ultrasound and computed tomography in rectal cancer: a meta-analysis. Colorectal Dis 2015;17:0129-35.

19. Ito K, Noro K, Yanagisawa Y, Sakamoto M, Mori S, et al. High-accuracy ultrasound contrast agent detection method for diagnostic ultrasound imaging systems. Ultrasound Med Biol 2015;41:3120-30.

20. Li L, Mori S, Kodama M, Sakamoto M, Takahashi S, et al. Enhanced sonographic imaging to diagnose lymph node metastasis: importance of blood vessel volume and density. Cancer Res 2013;73:2082-92.

21. Sato T, Takemura T, Ouchi T, Mori S, Sakamoto M, et al. Monitoring of blood vessel density using contrast-enhanced high frequency ultrasound may facilitate early diagnosis of lymph node metastasis. J Cancer 2017;8:704-15.

22. Iwaki T, Yamashita H, Hayakawa T. A color atlas of sectional anatomy of the mouse. Tokyo: Maruzen; 2015.

23. Fisher B, Fisher ER. Transmigration of lymph nodes by tumor cells. Science 1966;152:1397-8.

24. Fujii H, Horie S, Takeda K, Mori S, Kodama T. Optimal range of injection rates for a lymphatic drug delivery system. J Biophotonics 2018;11:e201700401.

25. Kodama T, Matsuki D, Tada A, Takeda K, Mori S. New concept for the prevention and treatment of metastatic lymph nodes using chemotherapy administered via the lymphatic network. Sci Rep 2016;6:32506.

26. Tada A, Horie S, Mori S, Kodama T. Therapeutic effect of cisplatin given with a lymphatic drug delivery system on false-negative metastatic lymph nodes. Cancer Sci 2017;108:2115-21.

27. Morton DL, Thompson JF, Cochran AJ, Mozzillo N, Nieweg OE, et al. Final trial report of sentinel-node biopsy versus nodal observation in melanoma. N Engl J Med 2014;370:599-609. 